\title{
Prospects for detecting light bosons at the FCC-ee and CEPC
}

\author{
We-Fu Chang, ${ }^{1,2, *}$ John N. Ng, ${ }^{2, \dagger}$ and Graham White ${ }^{2, *}$ \\ ${ }^{1}$ Department of Physics, National Tsing Hua University, Hsinchu 300, Taiwan \\ ${ }^{2}$ TRIUMF Theory Group, 4004 Wesbrook Mall, Vancouver, British Columbia V6T2A3, Canada
}

(Received 8 March 2018; published 11 June 2018)

\begin{abstract}
We look at the prospects for detecting light bosons, $X$, at proposed $\mathrm{Z}$ factories assuming a production of $10^{12} \mathrm{Z}$ bosons. Such a large yield is within the design goals of future FCC-ee and CEPC colliders. Specifically, we look at the cases where $X$ is either a singlet scalar that mixes with the standard model Higgs or a vector boson with mass $1 \lesssim M_{X} \lesssim 80 \mathrm{GeV}$. We find that several channels are particularly promising for discovery prospects. In particular, $Z \rightarrow f \bar{f} X$ and $Z \rightarrow V_{Q} X$ give a promising signal above a very clean standard model background. We also discuss several channels that have too large a background to be useful.
\end{abstract}

DOI: 10.1103/PhysRevD.97.115015

\section{INTRODUCTION}

So far, the LHC has not uncovered any unambiguous evidence for physics beyond the standard model(SM). It is striking then to consider that, despite this impressive advance at the energy frontier, the parameter space for new bosons that are lighter than a Higgs is still relatively unconstrained. This will remain true even in the case where the high luminosity LHC fails to find new physics [1]. Two proposed experiments, the FCC-ee and the CEPC[2-6], could either result in detection or falsify large parts of the parameter space for such a light boson. It is within the design goals of both experiments to produce up to $10^{12} \mathrm{Z}$ bosons per year, making the even rare decays probable. Such rare decays have been proposed as a way to constrain a hidden dark sector [7,8], as an indirect probe for supersymmetry [8], and as a probe to an electroweak phase transition [9].

In this work, we consider the singlet extension of the standard model as well as a new vector boson which couples to the standard model through effective operators. Such new bosons are ubiquitous in extensions to the standard model [1,10-22]. New scalar particles can be a dark matter candidate, a portal to the dark sector [23-31], as well as a catalyst for a strongly first-order electroweak phase transition [32-35], and a way to improve the stability of the vacuum [36-40]. We find that one can discover such a light scalar even for relatively small mixing angles of $\sin ^{2} \theta \sim 10^{-7} \rightarrow$ $10^{-3}$ with the SM Higgs boson depending on the mass and

\footnotetext{
*wfchang@phys.nthu.edu.tw

†misery@triumf.ca

gwhite@triumf.ca
}

Published by the American Physical Society under the terms of the Creative Commons Attribution 4.0 International license. Further distribution of this work must maintain attribution to the author(s) and the published article's title, journal citation, and DOI. Funded by SCOAP. the decay branching ratio $\operatorname{Br}(S \rightarrow$ final state). This impressive search power is due to the relatively clean SM backgrounds for decay channels $Z \rightarrow X f \bar{f}$ and $Z \rightarrow V_{Q} X$, where $V_{Q}$ is the $1^{3} S_{1}$ quarkonium with $Q \in(c, b)$. In both of these cases, the SM backgrounds are dominated by $X$ decaying into either $b \bar{b}, \mu \bar{\mu}$ or invisible final states (neutrinos, or massless Goldstone bosons, or yet unknown dark matter). Such channels turn out to be the most promising for reasons we discuss throughout the paper. We perform a systematic analysis of every possible decay channel of these types which we list in Table I. In the case of spin-1 boson extensions to the standard model, the limits we find depend on which fermion the boson couples to as well as its mass. The $\mathrm{Z}$ factories have the potential to directly detect a few $\mathrm{GeV}$ vector boson whose gauge coupling strength to the SM fermion is as small as $\sim 10^{-4} e$. Throughout this

TABLE I. Possible signals and their SM background. Fake signals within the SM are those whose invariant mass of the underlined fermion pair is close to $m_{X}$.

\begin{tabular}{lcc}
\hline \hline$Z$ decay & Subsequent $X$ decay & SM background \\
\hline$Z \rightarrow \nu_{i} \overline{\nu_{i}} X$ & $X \rightarrow b \bar{b}$ & $Z \rightarrow \underline{b \bar{b}}, \nu_{i} \bar{\nu}_{i}$ \\
& $X \rightarrow \mu \bar{\mu}$ & $Z \rightarrow \underline{\mu \bar{\mu}}, \nu_{i} \overline{\nu_{i}}$ \\
$Z \rightarrow b \bar{b} X$ & $X \rightarrow b \bar{b}$ & $Z \rightarrow \underline{b \bar{b}}, b \bar{b}$ \\
& $X \rightarrow \mu \bar{\mu}$ & $Z \rightarrow \underline{\mu \bar{\mu}}, b \bar{b}$ \\
$Z \rightarrow \mu \bar{\mu} X$ & $X \rightarrow$ invisible & $Z \rightarrow \underline{\nu_{i} \overline{\nu_{i}}}, b \bar{b}$ \\
& $X \rightarrow b \bar{b}$ & $Z \rightarrow \underline{b \bar{b}}, \mu \bar{\mu}$ \\
& $X \rightarrow \mu \bar{\mu}$ & $Z \rightarrow \underline{\mu \bar{\mu}}, \mu \bar{\mu}$ \\
$Z \rightarrow J / \psi X$ & $X \rightarrow$ invisible & $Z \rightarrow \underline{\nu_{i} \overline{\bar{\nu}_{i}}}, \mu \bar{\mu}$ \\
& $X \rightarrow \mu \bar{\mu}$ & $Z \rightarrow J / \psi \mu \bar{\mu}$ \\
$Z \rightarrow \Upsilon X$ & $X \rightarrow$ invisible & $Z \rightarrow J / \psi \underline{\nu_{i} \bar{\nu}_{i}}$ \\
& $X \rightarrow \mu \bar{\mu}$ & $Z \rightarrow \Upsilon \underline{\mu \bar{\mu}}$ \\
& $X \rightarrow$ invisible & $Z \rightarrow \Upsilon \underline{\nu_{i} \bar{\nu}_{i}}$ \\
\hline \hline
\end{tabular}


paper, both numerical and analytic methods are used with the numerical study relying heavily on the numerical package CALCHEP [41].

The structure of this paper is as follows. We discuss the singlet and spin-1 extensions of the standard model in Sec. II, then discuss the two most promising decay channels including an analysis of excesses over the SM expectations as a function of the parameter space and the standard model background in Secs. III and IV, respectively. Next, in Sec. V, we discuss the case where the singlet does not mix. Finally, in Sec. VI, we compare the signal to the background. We end with a conclusion.

\section{SCALAR AND SPIN-1 BOSON EXTENSIONS OF THE STANDARD MODEL}

Let us begin with a scalar singlet $(S)$ extension to the standard model. The singlet couples to the standard model through a Higgs portal term. A commonly used one is $S^{\dagger} S H^{\dagger} H$, where $H$ is the SM Higgs field. As such, the decay rates of $Z \rightarrow S f \bar{f}$ or $Z \rightarrow V_{Q} S$ only depend on the physical mass, $M_{S}$, of the singlet scalar and its mixing angle with the SM Higgs. $S$ is produced on shell and decays either visibly into SM particles or into some invisible final states. One will then observe a resonant peak in the invariant mass of the final states. Note that the invariant mass of the invisible decay can be well determined in the rest frame of the $\mathrm{Z}$ boson. The specific signal, $S \rightarrow Y$, is then controlled by the model-dependent branching ratio $\operatorname{Br}(S \rightarrow Y)$. We aim to calculate prospective limits in the $\sin ^{2} \theta \times \operatorname{Br}(S \rightarrow Y)$ vs $M_{S}$ plane at future $\mathrm{Z}$ factories. Note that these limits are independent of the details of the model.

To set up our conventions, we focus only on the relevant Lagrangian for a scalar field extension that is a singlet under the standard model gauge group and the interaction with the standard model is through the general Higgs portal. We denote the weak basis of the real components of SM Higgs and the singlet as $\left(h_{0}, s_{0}\right)$. For our purposes we will only need the mass squared matrix

$$
\left(\begin{array}{ll}
m_{h_{0}}^{2} & m_{s h}^{2} \\
m_{s h}^{2} & m_{s_{0}}^{2}
\end{array}\right) .
$$

We stress that the origins of the mass squared matrix is irrelevant to this work and it can arise from either explicit or spontaneous symmetry breaking. The mass matrix can be diagonalized by a rotation

$$
\left(\begin{array}{c}
H_{m} \\
S_{m}
\end{array}\right)=\left(\begin{array}{cc}
\cos \theta & -\sin \theta \\
\sin \theta & \cos \theta
\end{array}\right)\left(\begin{array}{c}
h_{0} \\
s_{0}
\end{array}\right)
$$

with mixing angle $\theta$, and

$$
\tan 2 \theta=\frac{2 m_{s h}^{2}}{m_{s_{0}}^{2}-m_{h_{0}}^{2}} .
$$

Here the range of the mixing angle is $\theta \in[-\pi / 2, \pi / 2]$. We consider the case where $m_{s_{0}}^{2}<m_{h_{0}}^{2}$ and the mass eigenvalues are

$$
\begin{gathered}
M_{H_{m}}^{2}=\frac{1}{2}\left[m_{h_{0}}^{2}+m_{s_{0}}^{2}+\sqrt{\left(m_{h_{0}}^{2}-m_{s_{0}}^{2}\right)^{2}+m_{s h}^{2}}\right], \\
M_{S_{m}}^{2}=\frac{1}{2}\left[m_{h_{0}}^{2}+m_{s_{0}}^{2}-\sqrt{\left(m_{h_{0}}^{2}-m_{s_{0}}^{2}\right)^{2}+m_{s h}^{2}}\right] .
\end{gathered}
$$

In the absence of mixing, i.e., when $m_{s h}^{2}=0$, one has $M_{H_{m}}=m_{h_{0}}$ and $M_{S_{m}}=m_{s_{0}}$.

$H_{m}$ is identified as the $M_{H}=125 \mathrm{GeV}$ SM-like Higgs boson and $S_{m}$ is the new neutral scalar boson with unknown mass $M_{S}$. For notational simplicity we shall drop the subscript $m$ for the mass eigenstates and use the shorthand notations $s_{\theta}, c_{\theta}$ for $\sin \theta, \cos \theta$. We are interested in the mass range $1 \lesssim M_{S} \lesssim 80 \mathrm{GeV}$. The upper bound is limited by kinematics and the lower bound of $M_{S} \simeq 1 \mathrm{GeV}$ is due to the energy resolution of the future $\mathrm{Z}$ factories which we assume to be around $1 \mathrm{GeV}$.

Finally we also consider the case of a new spin-1 boson with the same mass range. For simplicity, we only consider the case where the interaction Lagrangian between the spin1 boson and the standard model is phenomenologically parametrized as

$$
\mathcal{L} \supset\left(e g_{D}^{f}\right) \bar{f} \gamma_{\mu} f V_{D}^{\mu}
$$

The UV completion of such a model is not our concern in this work. See Refs. [42,43] for examples of plausible UV completions that give rise to the above operator.

In this case we have only two free parameters in the mass of the new boson and the coupling strength $g_{D}^{f}$ for each flavor. For the cases with axial vector couplings, the study can be easily extended with one more free parameter for each flavor. In general, the new vector boson could acquire flavor changing couplings. We will only focus on the case that the new vector boson couplings are flavor conserving. However, our study can be trivially extended to the flavor changing case where the SM background can be ignored and some useful new limits can be placed.

\section{DECAY CHANNEL $Z \rightarrow X f \bar{f}$}

In this section, we discuss the first promising decay channel where $X$ can be an invisible SM background $(\nu \bar{\nu})$, a visible SM background such as $f \bar{f}$ or the singlet particle we are searching for. For the case of fermions, we will only consider bottom quarks and muons, the former due to b-tagging and the latter due to its detectability arising from its long lifetime. Light jets and tau leptons turn out to be too noisy to compete with these channels. We will systematically study the decay rate as a function of the three parameters: the mass and the mixing angle (gauge coupling) of the light scalar (vector) as well as the decay branching fraction of $X$. 
In the case of the mixing angle (gauge coupling), the signal branching ratios are trivially proportional to $\sin ^{2} \theta\left(\left(g_{D}^{f}\right)^{2}\right)$ and we can simply divide by this quantity to give two parameter plots. The $Z$ can be produced almost at rest by precisely controlling the energies of $e^{ \pm}$beams. The invariant mass of $X$ can be determined by the squared of the 4-momentum sum of all its decay products. We are looking for a resonant invariant mass which peaks at $M_{X}$. On the other hand, even if $X$ decays invisibly; for example, in the process $Z\left(P_{Z}\right) \rightarrow f\left(k_{1}\right) \bar{f}\left(k_{2}\right) X\left(P_{X}\right)$, the invariant mass of $X$ can still be reconstructed by kinematics of the fermion pair in the $Z$ rest frame:

$$
\begin{aligned}
P_{X}^{2} & =\left[P_{Z}-\left(k_{1}+k_{2}\right)\right]^{2} \\
& =M_{Z}^{2}+M_{f f}^{2}-2 M_{Z}\left(E_{1}^{C M}+E_{2}^{C M}\right) .
\end{aligned}
$$

The SM $Z \rightarrow f \bar{f} f^{\prime} \bar{f}^{\prime}$ background is calculated by CALCHEP and summarized in Fig. 10.

In $e^{+} e^{-}$collisions at a resonance such as at the Z-pole, there is always an intrinsic nonresonance contribution to the final states. Most notably, when the final states involve charged fermion pairs, they can originate from virtual photon emissions from the initial states and t-channel processes with a very forward light fermion pair. We use CALCHeP to evaluate the full SM tree-level cross section $\sigma\left(e^{+} e^{-} \rightarrow\right.$ final states $)$, and we scan the c.m. energy within $M_{Z} \pm 10 \mathrm{GeV}$ to extract the continuous nonresonance SM background.

We found that the nonresonance background is indeed much smaller than the Z-resonance background. For example, the nonresonance to resonance ratios in $e^{+} e^{-} \rightarrow \mu \bar{\mu} b \bar{b}$ are $\{6.5,6.1,13.5\} \%$ for $b \bar{b}$ invariant mass $m_{b b}=$ $\{70 \pm 1,45 \pm 1,20 \pm 1\} \mathrm{GeV}$, and $\{4.3,8.3,10.5,17.0\} \%$ for muon pair $m_{\mu \mu}=\{70 \pm 1,45 \pm 1,20 \pm 1,10 \pm 1\} \mathrm{GeV}$. It is clear that the nonresonance contribution is larger for smaller $m_{\mu \mu}$ where virtual photon mediation is the main process. We obtained $\gtrsim 20 \%$ corrections from the nonresonant background for $m_{\mu \mu}<10 \mathrm{GeV}$ only. For cases with invisible final states such as $e^{+} e^{-} \rightarrow \nu \bar{\nu} b \bar{b}$ with $\{4.3,2.8,7.2\} \%$ for $b \bar{b}$ invariant mass $m_{b b}=\{70 \pm 1$, $45 \pm 1,20 \pm 1\} \mathrm{GeV}$, and $\{3.5,2.8,3.5\} \%$ for neutrino pair $m_{\nu \nu}=\{45 \pm 1,20 \pm 1,10 \pm 1\} \mathrm{GeV}$. Since the neutrino pair can only come from virtual $Z$, the nonresonance background is consistently small. Finally, we remark that the signal $X f \bar{f}$ can also have nonresonance contributions. However, these are typically much smaller than the contributions we calculated.

\section{A. $X=S$}

Let us begin with the case where $X$ is the light scalar for which we are ultimately searching. Due to the Yukawa suppression, we can ignore diagrams with $S$ attached to the fermion line. If $M_{S}>2 m_{b}$, the dominant decay channel is $S \rightarrow 2 b$. A useful kinetic variable $y_{b} \equiv m_{b b}^{2} / M_{Z}^{2}$ is defined, where $m_{b b}$ is the invariant mass of the $b \bar{b}$ pair. The on-shell light scalar gives a very narrow resonance peak in $y_{b}$ at around $y_{b}=\left(M_{S} / M_{Z}\right)^{2}$ and stands out from the continuous SM background. In this case, we can calculate the branching ratio analytically and find it to be

$\operatorname{Br}(Z \rightarrow S f \bar{f})=s_{\theta}^{2} \times F\left(M_{S} / M_{Z}\right) \times \operatorname{Br}(Z \rightarrow f \bar{f})$,

where

$$
\begin{aligned}
F(x)= & \frac{G_{F} M_{Z}^{2}}{24 \sqrt{2} \pi^{2}}\left[\frac{3 x\left(x^{4}-8 x^{2}+20\right)}{\sqrt{4-x^{2}}} \cos ^{-1}\left(\frac{x}{2}\left(3-x^{2}\right)\right)\right. \\
& \left.-3\left(x^{4}-6 x^{2}+4\right) \ln x-\frac{1}{2}\left(1-x^{2}\right)\left(2 x^{4}-13 x^{2}+47\right)\right],
\end{aligned}
$$

Using the PDG [44], we can acquire the relevant standard model branching ratios:

$$
\begin{aligned}
\operatorname{Br}(Z \rightarrow b \bar{b}) & =15.12 \%, \\
\operatorname{Br}\left(Z \rightarrow \nu_{i} \overline{\nu_{i}}\right) & =20.0 \%, \\
\operatorname{Br}(Z \rightarrow \mu \bar{\mu}) & =3.366 \% .
\end{aligned}
$$

In Fig. 1, we show the prediction for the $Z \rightarrow f \bar{f} S$ branching ratios normalized by $\sin ^{2} \theta$. In the case where the singlet mass is less than twice of the bottom mass, $M_{S}<2 m_{b}$, the cleanest signal will be $S \rightarrow e e, \mu \mu$ which can be used to reconstruct the $S$ resonance using the quantities $y_{e}$ and $y_{\mu}$.

For the case of $S$ decaying into invisible final states, the SM background is $Z \rightarrow \nu \bar{\nu} f \bar{f}$. As discussed before, the invariant mass of $X$ can still be reconstructed from the other visible fermions since the $Z$ boson can be produced nearly at rest.

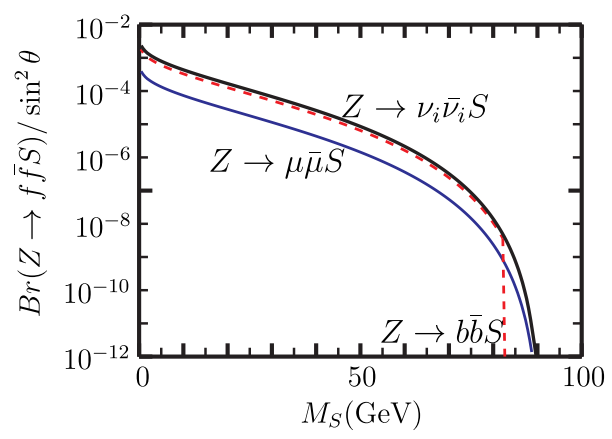

FIG. 1. The decay branching ratios of $Z \rightarrow f \bar{f} S$ over the mixing angle squared vs $M_{S}$. The red solid/blue dash/black line is for $f=\mu / b / \nu_{i}$. 


\section{B. $X=V_{D}$}

The decay width of $V_{D} \rightarrow f f$ can be derived to be

$$
\Gamma_{V_{D} \rightarrow f f}=\frac{N_{c}^{f} \alpha\left(g_{D}^{f}\right)^{2}}{3} M_{V_{D}}\left(1+2 r_{f}^{2}\right) \sqrt{1-4 r_{f}^{2}},
$$

where $r_{f}=m_{f} / M_{V_{D}}$. Note that there is no tree-level $V_{D} Z Z$ coupling and the form factor has to be symmetrized for the two Z's. By labeling the process as $Z(q, \mu) \rightarrow$ $f\left(p_{1}\right) \bar{f}\left(p_{2}\right) V_{D}(k, \nu)$, where the $V_{D}$ is attached to the fermion line, the amplitude reads

$$
\begin{aligned}
i \mathcal{M}= & -i \bar{u}\left(p_{1}\right)\left[\frac{-\not k \gamma^{\nu} \gamma^{\mu}+2 p_{1}^{\nu} \gamma^{\mu}}{m_{V_{D}}^{2}+2 p_{1} \cdot k}-\frac{-\gamma^{\mu} \gamma^{\nu} \not k+2 p_{2}^{\mu} \gamma^{\nu}}{m_{V_{D}}^{2}+2 p_{2} \cdot k}\right] \\
& \times\left(\frac{e g_{2} g_{D}^{f}}{c_{W}}\right)\left(g_{V}^{f}-g_{A}^{f} \gamma^{5}\right) v\left(p_{2}\right) \epsilon_{\nu}^{*}(k) \epsilon_{\mu}(q),
\end{aligned}
$$

after applying the equation of motions and $(\epsilon \cdot k)=0$. Here, $g_{A}^{f}=T_{3}^{f} / 2$ and $g_{V}=T_{3}^{f} / 2-q^{f} s_{W}^{2}$ are the SM Z couplings. The complete analytic expression for the cross section is too complicated for practical use. For $f \neq b$, the $m_{f}=0$ limit is a good approximation, and the simplified differential decay width reads

$$
\begin{aligned}
\frac{d^{2} \Gamma_{Z \rightarrow V_{D} f \bar{f}}}{d x d y}= & \frac{\alpha\left(g_{D}^{f}\right)^{2} G_{F} M_{Z}^{3}}{3 \sqrt{2} \pi^{2}} \frac{N_{c}^{f}\left[\left(g_{V}^{f}\right)^{2}+\left(g_{A}^{f}\right)^{2}\right]}{y^{2}(1+d-x-y)^{2}} \\
& \times\left[4 y^{3}(1+d-x)+y(1+d-x)\right. \\
& \times\left(1+x^{2}+d^{2}+4 d\right)-2 y^{4}-d(1+d-x)^{2} \\
& \left.-y^{2}\left(3+3 d^{2}+3 x^{2}+8 d-4 x-4 x d\right)\right],
\end{aligned}
$$

where $d=\left(M_{V_{D}} / M_{Z}\right)^{2}, x \equiv\left(p_{1}+p_{2}\right)^{2} / M_{Z}^{2}$, and $y \equiv$ $\left(p_{2}+k\right)^{2} / M_{Z}^{2}$. The kinematics are $0 \leq x \leq(1-\sqrt{d})^{2}$ and $(1+d-x) / 2-\lambda_{c m}(x, d) / 2 \leq y \leq(1+d-x) / 2+\lambda_{c m}(x, d) / 2$, where $\lambda_{c m}\left(x_{1}, x_{2}\right) \equiv \sqrt{1+x_{1}^{2}+x_{2}^{2}-2\left(x_{1}+x_{2}+x_{1} x_{2}\right)}$. The $y$ integration can be performed analytically, but we do not find a closed form expression for the double integration. Instead, we will just evaluate the complete differential decay rate, with $m_{f} \neq 0$, numerically.

The widths of $Z \rightarrow V_{D} f \bar{f}$ are displayed in Fig. 2.

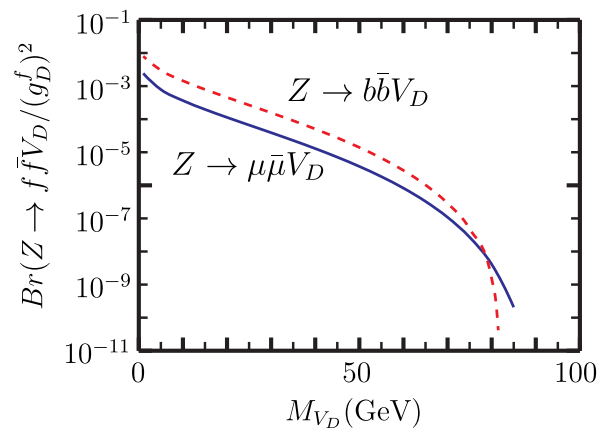

FIG. 2. The decay branching ratio of $Z \rightarrow f f V_{D}$ over the coupling strength squared vs $M_{V_{D}}$.

\section{DECAY CHANNEL $Z \rightarrow V_{Q} X$}

Before considering the process $Z \rightarrow V_{Q} X$, we shall study the SM background $Z \rightarrow V_{Q} f \bar{f},(f=\mu, \nu)$. For simplicity, we will not consider $f=c, b$, which are complicated by QCD. We need to derive the $Z Z V_{Q}$ coupling vertex for calculating the SM process $Z \rightarrow V_{Q} \nu_{i} \bar{\nu}_{i}$. Another SM background $Z \rightarrow \nu \bar{\nu} Z^{*}\left(Z^{*} \rightarrow V_{Q}\right)$ is negligible. Following [45], the desired vertex can be derived from the Feynman diagrams; see Fig. 3. Labeling the momenta and polarizations as $Z\left(p_{1}, \epsilon_{1}\right)-Z\left(p_{2}, \epsilon_{2}\right)-V_{Q}\left(P_{V}, \epsilon_{V}\right),\left(p_{1}+p_{2}=p_{V}\right)$, the reduced amplitude (without the quark spinor wave functions) reads

$$
\begin{aligned}
i \widetilde{\mathcal{M}} & =i \frac{g_{2}^{2} / c_{W}^{2}}{\left(p_{1} \cdot p_{2}\right)}\left[\phi_{2}\left(g_{V}^{Q}-g_{A}^{Q} \gamma^{5}\right)\left(q_{1}-\not p_{2}+m_{Q}\right) \phi_{1}\left(g_{V}^{Q}-g_{A}^{Q} \gamma^{5}\right)\right. \\
& \left.+\phi_{1}\left(g_{V}^{Q}-g_{A}^{Q} \gamma^{5}\right)\left(\not \not_{2}-q_{2}+m_{Q}\right) \phi_{2}\left(g_{V}^{Q}-g_{A}^{Q} \gamma^{5}\right)\right],
\end{aligned}
$$

where $g_{V}^{Q}$ and $g_{A}^{Q}$ are the SM heavy quark-Z couplings. In the NR limit, $q_{1} \sim q_{2} \sim p \equiv p_{V} / 2$ and $q_{1}^{2} \sim q_{2}^{2} \sim m_{Q}^{2}$. Including the spin projection and the quarkonium wave function, the coupling is

$$
\begin{aligned}
i \mathcal{M} \simeq- & \frac{\sqrt{N_{c} m_{V}} \phi_{0}^{Q}}{2 m_{Q}} \operatorname{Tr}\left[i \tilde{\mathcal{M}} \phi_{V}^{*}\left(\not p+m_{Q}\right)\right] \\
= & \frac{A_{Z Z V}^{Q}}{\left(p_{1} \cdot p_{2}\right)} \epsilon^{\epsilon_{V}, \epsilon_{1}, \epsilon_{2}, p_{1}-p_{2}}
\end{aligned}
$$

where $\phi_{0}^{Q}$ is the wave-function at the origin for $V_{Q \bar{Q}}$, and

$$
A_{Z Z V}^{Q}=8\left(\frac{g_{2}}{c_{W}}\right)^{2} \sqrt{3 m_{V}} \phi_{0}^{Q} g_{A}^{Q} g_{V}^{Q} .
$$

Note that the vertex admits the Bose symmetry under $Z\left(p_{1}, \epsilon_{1}\right) \Leftrightarrow Z\left(p_{2}, \epsilon_{2}\right)$ exchange. By using the following values [44,46], $M_{J / \psi}=3.0969 \mathrm{GeV}, M_{\Upsilon}=9.4603 \mathrm{GeV}$, $\phi_{0}^{J / \psi}=0.270(20)(\mathrm{GeV})^{3 / 2}$, and $\phi_{0}^{\Upsilon}=0.715(24)(\mathrm{GeV})^{3 / 2}$, we have $A_{Z Z V}^{c}=0.0866(64)(\mathrm{GeV})^{2}$ and $A_{Z Z V}^{b}=$ $0.723(24)(\mathrm{GeV})^{2}$.

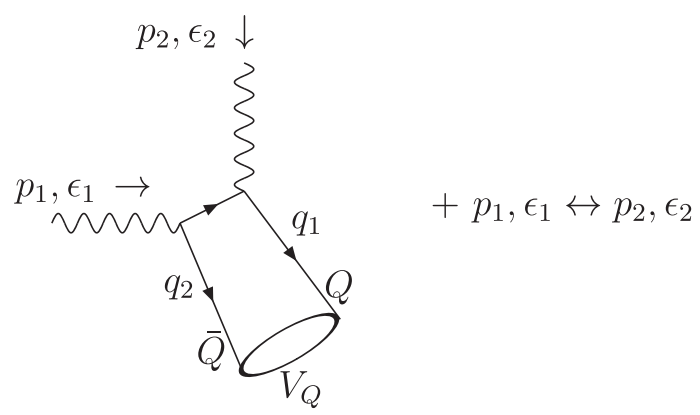

FIG. 3. The Feynman diagrams for the $Z Z V_{Q}$ coupling. 
Now we can calculate the SM background $Z\left(P_{Z}\right) \rightarrow$ $V_{Q}\left(P_{V}\right) \nu\left(k_{1}\right) \bar{\nu}\left(k_{2}\right)$. The differential decay width can be calculated to be

$$
\frac{d \Gamma_{Z \rightarrow V_{Q} \nu \nu}}{d x}=\frac{1}{192 \pi^{3} \sqrt{2}} \frac{\left|A_{Z Z V}\right|^{2} G_{F}}{M_{Z} v_{Q}} F_{v \nu \nu}\left(x, v_{Q}\right),
$$

where $v_{Q} \equiv\left(M_{V_{Q}} / M_{Z}\right)^{2}, x \equiv\left(k_{1}+k_{2}\right)^{2} / M_{Z}^{2}$ and we have summed over all three neutrino species. The function $F_{v v \nu}$ is given by

$$
\begin{aligned}
F_{v \nu \nu}\left(x, v_{Q}\right)= & \frac{\lambda_{c m}\left(x, v_{Q}\right)}{(1-x)^{2}\left(1+x-v_{Q}\right)^{2}} \\
& \times\left[v_{Q}^{3}(1+x)-2 v_{Q}^{2}\left(1+6 x+x^{2}\right)\right. \\
& \left.+v_{Q}\left(1+15 x+15 x^{2}+x^{3}\right)+4 x(1-x)^{2}\right] .
\end{aligned}
$$

The total branching ratios can be evaluated to be

$$
\begin{gathered}
\operatorname{Br}\left(Z \rightarrow J / \psi \nu_{i} \bar{\nu}_{i}\right)=(1.29 \pm 0.10) \times 10^{-11}, \\
\operatorname{Br}\left(Z \rightarrow \Upsilon \nu_{i} \bar{\nu}_{i}\right)=(0.96 \pm 0.07) \times 10^{-10},
\end{gathered}
$$

where the uncertainty arises from the wave function of quarkonium. Again, even though the neutrinos are invisible, the invariant mass squared of two neutrinos can be reconstructed by the energy of the quarkonium in the rest frame of $Z$ :

$$
x M_{Z}^{2}=\left(k_{1}+k_{2}\right)^{2}=\left(P_{Z}-P_{V}\right)^{2}=M_{Z}^{2}+M_{V}^{2}-2 M_{Z} E_{V}^{C M} .
$$

This fakes the signal of $Z \rightarrow X V_{Q}$ production with subsequent invisible $X$ decays.

For $Z \rightarrow V_{Q} X$ with mass $m_{X}$, the SM background is, therefore,

$$
\Delta \Gamma_{Z \rightarrow V_{Q} \nu \bar{\nu}}^{\mathrm{SM}}\left(m_{X}\right)=\int_{\left(m_{X}-\delta E\right)^{2} / M_{Z}^{2}}^{\left(m_{X}+\delta E\right)^{2} / M_{Z}^{2}}\left(\frac{d \Gamma_{V \nu \nu}}{d x}\right) d x
$$

for a yet unknown detector-dependent invariant mass resolution, $\delta E$. We take $\delta E \sim 1 \mathrm{GeV}$ as a conservative guess of the energy resolution at the future $\mathrm{Z}$ factory, the result is displayed in Fig. 4.

Next, we turn our attention to the SM background for the signal $Z \rightarrow V_{Q} X$ where $X$ decays into $\mu \bar{\mu}$. In this case one looks for the resonance peak of $m_{\mu \bar{\mu}}^{2}=M_{X}^{2}$. For $Z \rightarrow V_{Q} X(X \rightarrow \mu \bar{\mu})$, the dominant SM background comes from $Z \rightarrow V_{Q} \gamma^{*}$ and the virtual photon turns into a muon pair. Similar to what we did for the $Z Z V_{Q}$, just replacing one $Z$ by a photon, the SM "direct" $Z-\gamma-V_{Q}$ coupling (amplitude) is worked out to be

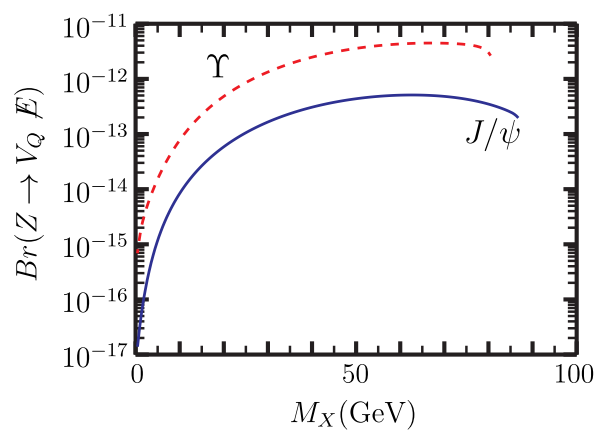

FIG. 4. The SM background for $Z \rightarrow V_{Q}+X$ vs $M_{X}$ with a projective $1 \mathrm{GeV}$ energy resolution at the $\mathrm{Z}$ factory, where $X$ refers to $S$ or $V_{D}$, and $X$ decays invisibly. The blue/red curve is for $Z \rightarrow\left(J / \psi \nu_{i} \bar{\nu}_{i}\right) /\left(\Upsilon \nu_{i} \bar{\nu}_{i}\right)$

$$
\mathcal{M}=A_{\gamma}^{Q} \epsilon^{\epsilon_{Z}, \epsilon_{\gamma}^{*}, \epsilon_{V}^{*}, p_{\gamma}}
$$

with the dimensionless coupling

$$
A_{\gamma}^{Q}=8 i e_{Q} g_{A}^{Q}\left(\frac{e g_{2}}{c_{W}}\right) \frac{\sqrt{N_{c} M_{V}} \phi_{0}^{Q}}{M_{Z}^{2}\left(1-v_{Q}\right)} .
$$

Note that there is also a loop induced "indirect" contribution to this process [46,47]. However its contribution is small, $\sim-5 \%$ of the direct contribution and we thus ignore it in this work.

Plugging in the numbers, we have $A_{\gamma}^{c}=3.05(23) \times 10^{-5}$ and $A_{\gamma}^{b}=7.14(24) \times 10^{-5}$ for $J / \Psi$ and $\Upsilon$, respectively. The width of 2-body $Z$ decay $Z \rightarrow f_{1} f_{2}$ is $\Gamma=$ $p_{c m}\left\langle|\mathcal{M}|^{2}\right\rangle / 8 \pi M_{Z}^{2}$, where $p_{c m}=\frac{M_{Z}}{2} \lambda_{c m}\left(x_{1}, x_{2}\right)$ is the final state particle 3-momentum in the rest frame, and $x_{i} \equiv\left(m_{i} / M_{Z}\right)^{2}$. Then, the total decay width of can be calculated to be

$$
\Gamma_{Z \rightarrow \gamma V_{Q}}=\frac{\left|A_{\gamma}^{Q}\right|^{2} M_{Z}}{96 \pi} \frac{\left(1+v_{Q}\right)\left(1-v_{Q}\right)^{3}}{v_{Q}} .
$$

Note that these results agree with [45]. With the $Z \gamma V_{Q}$ vertex in hand, we can now consider the case that $X$ decays visibly, into $\mu \bar{\mu}$. The differential width of $Z \rightarrow$ $V_{Q} \mu\left(k_{1}\right) \bar{\mu}\left(k_{2}\right)$ is straightforwardly calculated to be

$$
\frac{d \Gamma_{Z \rightarrow V_{Q} \mu \mu}}{d x}=\frac{\alpha\left|A_{\gamma}^{Q}\right|^{2} M_{Z}}{288 \pi^{2} v_{Q}} F_{V \mu}\left(x, f_{\mu}, v_{Q}\right)
$$

where the dimensionless variables are defined as $\left(k_{1}+k_{2}\right)^{2}=x M_{Z}^{2}, f_{\mu} \equiv\left(m_{\mu} / M_{Z}\right)^{2}$, and 


$$
\begin{aligned}
F_{V \mu}(x, f, v)= & \frac{\lambda_{c m}(x, v)}{x^{2}}\left\{3 f^{2}(1+v) x\right. \\
& +3 f\left[v^{3}-v^{2}(1+2 x)+v\left(x^{2}+4 x-1\right)\right. \\
& \left.+(1-x)^{2}\right]+x\left[v^{3}-v^{2}(1+2 x)\right. \\
& \left.\left.+v\left(x^{2}+8 x-1\right)+(1-x)^{2}\right]\right\} .
\end{aligned}
$$

The range of $x$ for the massive fermion final state is now $4 f_{\mu} \leq x \leq\left(1-\sqrt{v_{Q}}\right)^{2}$. The total branching ratios can be numerically evaluated to be

$$
\begin{gathered}
\operatorname{Br}(Z \rightarrow J / \psi \mu \bar{\mu})=(8.97 \pm 1.37) \times 10^{-10}, \\
\operatorname{Br}(Z \rightarrow \Upsilon \mu \bar{\mu})=(5.15 \pm 0.35) \times 10^{-10} .
\end{gathered}
$$

Once again the uncertainties in the above arise from the wave function of quarkonium. Because of the photon mediation, these two branching ratios are roughly 2 orders of magnitude larger than the previously calculated $\operatorname{Br}\left(Z \rightarrow V_{Q} \nu_{i} \bar{\nu}_{i}\right)$. Similar to before, we integrate over the SM differential cross section at the vicinity of $x=$ $\left(M_{X} / M_{Z}\right)^{2}$ with the detector energy resolution $\delta E$ which we assume to be around $\sim 1 \mathrm{GeV}$. The $\mathrm{SM}$ background for $Z \rightarrow V_{Q} X(X \rightarrow \mu \bar{\mu})$ is

$$
\Delta \Gamma_{Z \rightarrow V_{Q} \mu \bar{\mu}}^{\mathrm{SM}}\left(m_{X}\right)=\int_{\left(m_{X}-\delta E\right)^{2} / M_{Z}^{2}}^{\left(m_{X}+\delta E\right)^{2} / M_{Z}^{2}}\left(\frac{d \Gamma_{Z \rightarrow V_{Q} \mu \mu}}{d x}\right) d x .
$$

Due to the photon propagator, the differential rate peaks at small $x$ (see Fig. 5). The SM background drops rapidly as $X$ gets heavier rendering the SM background basically irrelevant for $M_{X}>60 \mathrm{GeV}$.

\section{A. $X=V_{D}$}

The $Z V_{Q} V_{D}$ coupling can be easily extended from the $\mathrm{SM} Z V_{Q} \gamma$ vertex. For the case of a light vector, we can just

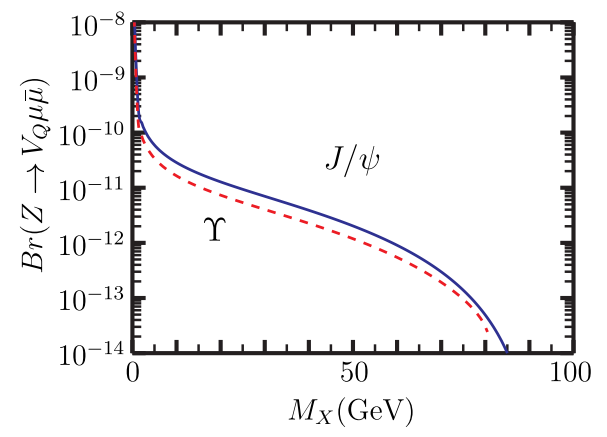

FIG. 5. The SM background for $Z \rightarrow V_{Q}+X$ vs $M_{X}$ with a projective $1 \mathrm{GeV}$ energy resolution at the $\mathrm{Z}$ factory, where $X$ refers to $S$ or $V_{D}$, and $X$ decays into $\mu \bar{\mu}$. The blue/red curve is for $Z \rightarrow(J / \psi \mu \bar{\mu}) /(\Upsilon \mu \bar{\mu})$.

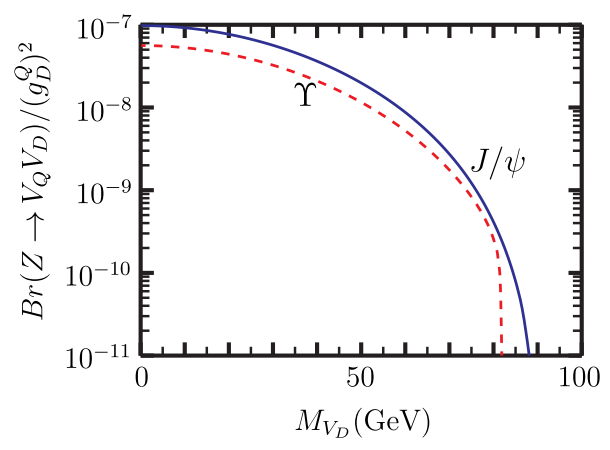

FIG. 6. The branching ratio over $g_{D}^{2}$ vs $M_{V_{D}}$. The blue/red curve is for $Z \rightarrow\left(J / \psi V_{D}\right) /\left(\Upsilon V_{D}\right)$.

multiply $A_{\gamma}$ by $g_{D}^{Q}$ and take the light vector mass into account,

$$
A_{\gamma_{D}}=i 8 g_{D}^{Q} e_{Q} g_{A}^{Q}\left(\frac{e g_{2}}{c_{W}}\right) \frac{\sqrt{N_{c} M_{V}} \phi_{0}^{Q}}{M_{Z}^{2}\left(1-v_{Q}+d\right)},
$$

where $d=\left(M_{V_{D}} / M_{Z}\right)^{2}$. The decay width becomes

$$
\begin{aligned}
\Gamma_{Z \rightarrow V_{Q} V_{D}}= & \frac{\left(g_{D}^{Q}\right)^{2}\left|A_{\gamma}\right|^{2} M_{Z}}{96 \pi v_{Q}} \times\left(\frac{1-v_{Q}}{1+d-v_{Q}}\right)^{2} \times \lambda_{c m}\left(v_{Q}, d\right) \\
& \times\left\{\left(1+v_{Q}\right)\left[\left(1-v_{Q}\right)^{2}+d^{2}\right]\right. \\
& \left.-2 d\left(1-4 v_{Q}+v_{Q}^{2}\right)\right\}
\end{aligned}
$$

which is proportional to $\left(g_{D}^{Q}\right)^{2}$. The $Z \rightarrow V_{Q} V_{D}$ decay branching ratio modulated the unknown $V_{D}$ coupling $\left(g_{D}^{Q}\right)^{2}, Q=c, b$ is shown in Fig. 6 .

\section{B. $X=S$}

For $Z\left(P_{Z}, \epsilon_{Z}\right) \rightarrow V_{Q}\left(P_{V}, \epsilon_{V}\right) S$, there are three Feynman diagrams we need to consider; see Fig. 7 . With the same token, the $Z S V_{Q}$ vertex can be calculated to be

$$
s_{\theta} A_{s}^{Q} M_{Z}\left[-\frac{\epsilon_{V} \cdot \epsilon_{Z}}{1-v_{Q}}+\frac{P_{V} \cdot P_{Z} \epsilon_{V} \cdot \epsilon_{Z}-P_{V} \cdot \epsilon_{Z} P_{Z} \cdot \epsilon_{V}}{M_{Z}^{2}\left(1+s-v_{Q}\right)}\right]
$$
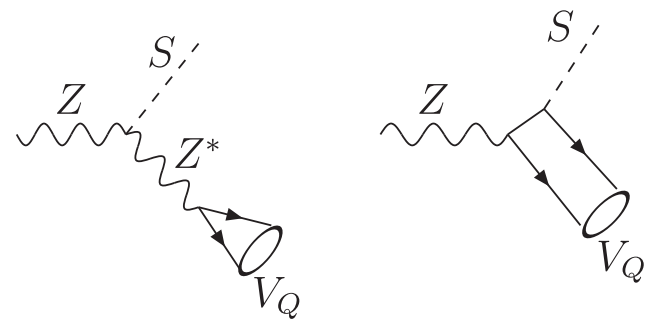

FIG. 7. The Feynman diagrams for the $Z S V_{Q}$ coupling. Note that there are two ways of connecting the $S$ to the fermion lines. 


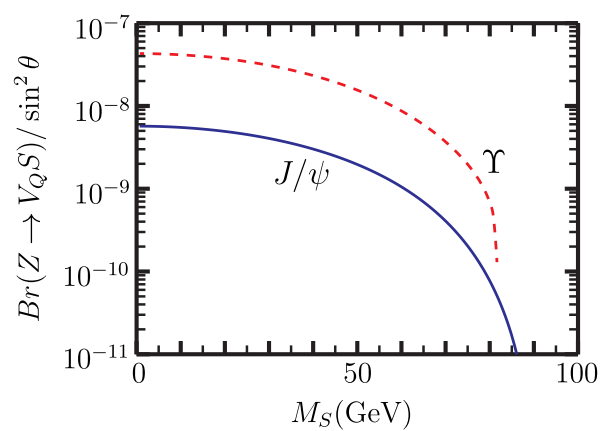

FIG. 8. The branching ration over $s_{\theta}^{2}$ vs $M_{S}$. The blue/red curve is for $Z \rightarrow(J / \psi S) /(\Upsilon S)$.

with the dimensionless coupling

$$
A_{s}^{Q}=2\left(\frac{g_{2}}{c_{W}}\right)^{2} \frac{\sqrt{N_{c} M_{V}} g_{V}^{Q} \phi_{0}^{Q}}{M_{Z}^{2}},
$$

where $s \equiv \frac{M_{S}^{2}}{M_{Z}^{2}}$. For $J / \Psi$ and $\Upsilon, A_{s}^{c}=1.04(8) \times 10^{-5}$ and $A_{s}^{b}=-8.69(29) \times 10^{-5}$, respectively. We have

$$
\begin{aligned}
\Gamma_{Z \rightarrow V_{Q} S}= & \frac{\left|A_{s}\right|^{2} s_{\theta}^{2} M_{Z}}{192 \pi} \frac{\lambda_{c m}\left(v_{Q}, s\right)}{v_{Q}}\left\{\frac{\left(1+v_{Q}-s\right)^{2}+8 v_{Q}}{\left(1-v_{Q}\right)^{2}}\right. \\
& -\frac{12 v_{Q}\left(1+v_{Q}-s\right)}{\left(1-v_{Q}\right)\left(1+s-v_{Q}\right)} \\
& \left.+\frac{2 v_{Q}\left[\left(1+v_{Q}-s\right)^{2}+2 v_{Q}\right]}{\left(1+s-v_{Q}\right)^{2}}\right\}
\end{aligned}
$$

The first term in the curvy bracket represents the contribution of the diagram where $S$ connects to the $\mathrm{Z}$ boson. The third term represents the contributions where $S$ connects to the quark lines in the quarkonium, and the middle term is the interference contribution. Note that, $s_{\theta}$ aside, there is a sign difference, compared with [45], for the first $\epsilon_{V} \cdot \epsilon_{Z}$ term in Eq. (4.22) due to a difference in convention. However, for the decay width, Eq. (4.23) agrees with [45].

$\operatorname{The} \operatorname{Br}\left(Z \rightarrow S V_{Q}\right) / s_{\theta}^{2}$ is displayed in Fig. 8. Note that the $\operatorname{Br}(Z \rightarrow \Upsilon S)$ is about 1 order of magnitude larger than $\operatorname{Br}(Z \rightarrow J / \psi S)$. This can be understood due to the ratio $\left(g_{V}^{b} / g_{V}^{c}\right)^{2} \cdot\left(M_{\Upsilon} / M_{J / \psi}\right) \cdot\left(m_{b} / m_{c}\right)^{3} \sim 10$.

\section{WHEN $S$ DOES NOT MIX}

Let us briefly consider the case where $S$ does not develop a vacuum expectation value(VEV). In this case, the only coupling to the standard model is through the interaction with the Higgs. After spontaneous symmetry breaking, we have a dimensionful coupling between the singlet and the Higgs which controls the decay rate. The relevant interaction is parametrized as

$$
\frac{\kappa v_{H}}{2} H S^{2}
$$

where $v_{H}=246 \mathrm{GeV}$ is the VEV of the SM Higgs. Then the $H \rightarrow 2 S$ decay width can be calculated as

$$
\Gamma_{H \rightarrow 2 S}=\frac{\kappa^{2} v_{H}^{2}}{32 \pi M_{H}} \sqrt{1-\left(4 M_{S}^{2} / M_{H}^{2}\right)}
$$

The above contributes to the Higgs invisible decay. ATLAS [48] and CMS [49] give limits on the Higgs invisible decay of $B r_{i n v}<0.28$ and $B r_{i n v}<0.36$ at 95\% CL, respectively. Using the ATLAS bound, it amounts to $\Gamma_{\text {inv }}<0.388 \Gamma_{\text {visible }}=1.58 \mathrm{MeV}$ for the $\mathrm{SM} 125 \mathrm{GeV}$ Higgs. This translates to an upper bound on the triple scalar coupling of

$$
\kappa^{2}<\frac{1}{\sqrt{1-4 M_{S}^{2} / M_{H}^{2}}} \frac{32 \pi M_{H} \Gamma_{\mathrm{inv}}}{v_{H}^{2}} .
$$

The coupling is severely constrained to be smaller than $\lesssim 4 \times 10^{-4}$ for the mass range we are interested in, $M_{S}<M_{H} / 2$. The widths of $Z \rightarrow \mu \bar{\mu} S S$ can be calculated by CalcHeP. For $M_{S}=5(20) \mathrm{GeV}$, the width is $2.3 \times 10^{-11}\left(2.01 \times 10^{-12}\right) \times\left(\kappa^{2} / 10^{-4}\right) \mathrm{GeV}$. And the SM background will be $Z \rightarrow \mu \bar{\mu} \nu \bar{\nu}$ and the width is $2.662 \times 10^{-8} \mathrm{GeV}$. Given $10^{12} \mathrm{Z}$ bosons, the number of expected signal events is around $\mathcal{O}(1)$ and the expected number background events is around $10^{4}$. Hence, the $Z$ factory cannot compete with the Higgs invisible decay constraint in this scenario.

Similar excise can be carried out for the Higgs factory. We find the signal to background ratio is smaller than $10^{-6}$. Moreover, even with a luminosity around $a b^{-1}$, the expected signal event number is $\lesssim \mathcal{O}(1)$.

\section{RESULTS AND CONCLUSIONS}

In this work, we have studied the possibility of probing the parameter space for the light boson, $X$, extensions to the standard model with a $Z$ factory. We have focused on the rare $Z$ decays $Z \rightarrow f \bar{f} X$ and $Z \rightarrow V_{Q} X$, where the fermions are either invisible states, $b$ quarks, or muons. These states are useful probes due to the increasing efficiency of $b$ tagging and relatively long lifetime, respectively. Other channels are less useful for such a search. In particular, the light jets have a noisy background, and the $\tau$ lepton has multiple hadronic decay channels rendering it more difficult to reconstruct. Moreover, our formulas can be easily extended to these cases. The SM 4-body $Z$ decay backgrounds listed in Table I are evaluated by CALCHEP and displayed in Fig. 10.

For the $Z \rightarrow V_{Q} X$ background $Z \rightarrow V_{Q} f \bar{f}$, with $f=\mu$, $\nu$, we derived the analytic expressions and the numerical evaluation of these expressions are displayed in Fig. 4 and Fig. 5. To avoid complications arising from QCD, we do not consider the channel $Z \rightarrow V_{Q} b \bar{b}$.

We now have all the ingredients to compare signals with SM backgrounds. In Fig. 9, we plot the curves corresponding to the $3 \sigma$ limit that satisfies 


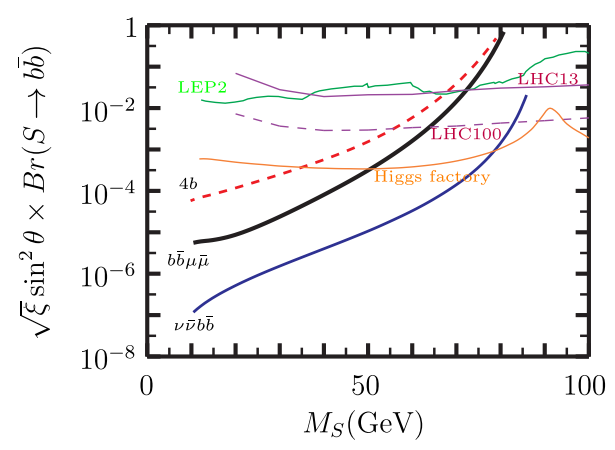

(a)

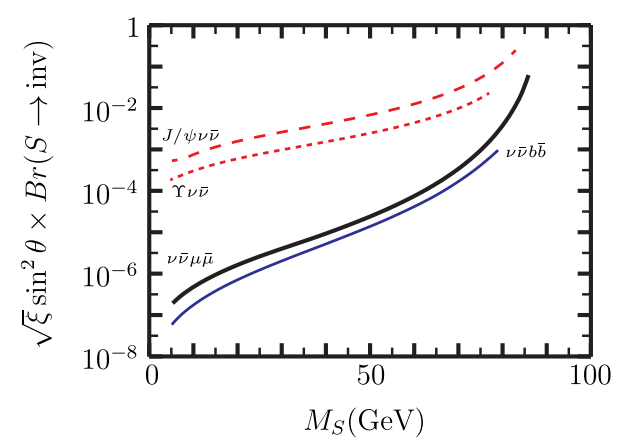

(c)

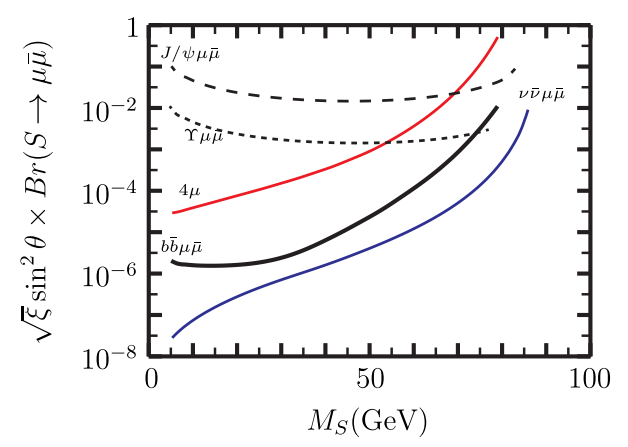

(e)

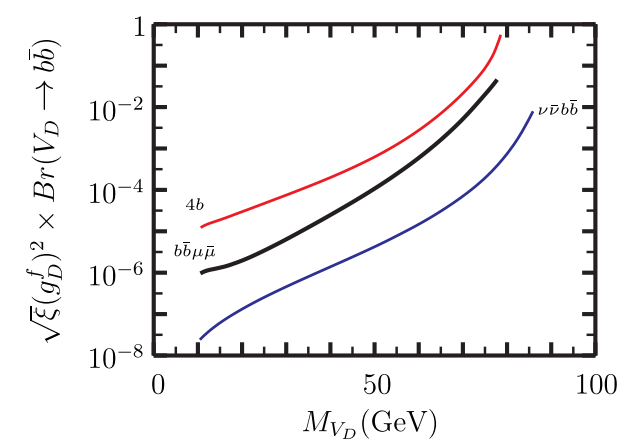

(b)

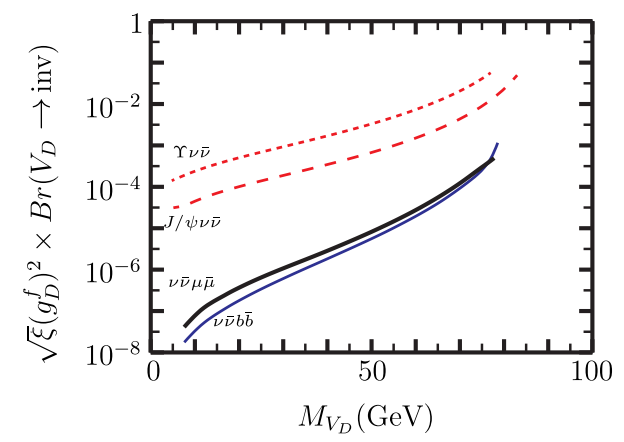

(d)

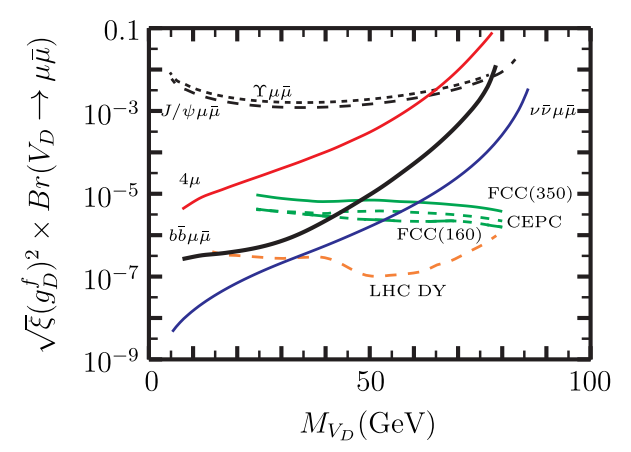

(f)

FIG. 9. (a), (c), (e) The $3 \sigma$ limits on $\sqrt{\xi} s_{\theta}^{2} \times \operatorname{Br}(S \rightarrow f \bar{f})$ vs $M_{S}$ with $10^{12} Z$ 's. (b,d,f) The $3 \sigma$ limits on $\sqrt{\xi}\left(g_{D}^{f}\right)^{2} \times \operatorname{Br}\left(V_{D} \rightarrow f^{\prime} \bar{f}^{\prime}\right)$ vs $M_{V_{D}}$ with $10^{12} Z$ 's and energy resolution $\triangle E=1 \mathrm{GeV}$. Here $\xi$ denotes the unknown total detection efficiencies for the signals. In (a), we also display the limits from the direct search at LEP2 [50](where the detection efficiencies have been taken into account), $p p \rightarrow$ $t \bar{t} S(b \bar{b})$ at HLC13 and HLC100 with $\mathcal{L}=3 \mathrm{ab}^{-1}$, and at the Higgs factory [1] with $\mathcal{L}=1 \mathrm{ab}^{-1}$. In (f), some limits adopted from the Drell-Yan process at LHC [51] and $e^{+} e^{-} \rightarrow \gamma \mu \bar{\mu}$ at the CEPC and FCC [52] are displayed. Note, however, that these limits apply to the kinetic $U(1)_{Y}-U(1)_{\text {hidden }}$ mixing model only.

$\frac{S}{\sqrt{B}}=\frac{\operatorname{Br}(\text { signal }) \times 10^{6} \times \sqrt{\xi}}{\sqrt{\operatorname{Br}(\text { background, } \triangle E=1 \mathrm{GeV}) \times(\triangle E / 1 \mathrm{GeV})}}=3$,

with $10^{12}$ fiducial $Z$ events and final state invariant mass resolution $\triangle E=1 \mathrm{GeV} .{ }^{1}$ We do not include the uncertainty in the quarkonium wave function, $\sim \mathcal{O}(10 \%)$, for the

\footnotetext{
${ }^{1}$ The expected precision of invariant mass at the planning Z-factories (see Fig. 3.15 and Fig. 3.16 in [2]) are about $\delta m_{b \bar{b}} \sim$ $2 \mathrm{GeV}$ and $\delta m_{\mu \bar{\mu}} \sim 0.2 \mathrm{GeV}$ for the $b \bar{b}$ and $\mu \bar{\mu}$, respectively.
}

processes involving $V_{Q}$, nor the nonresonant SM backgrounds, $\sim \mathcal{O}(10 \%)$, as their effects are barely visible in the figure. Here we also included the unknown overall detection efficiency $\xi$ for a specific channel. For those channels with SM background branching ratios smaller than $10^{-12}$, we use $10^{-12}$ as the background.

When the energy resolution is reasonably small, the number of background events is linearly proportional to the energy resolution. For a different energy resolution, the corresponding $3 \sigma$ limits can be easily read from Fig. 9 with a vertical shift $\frac{1}{2} \log _{10}(\triangle E / 1 \mathrm{GeV})$. 


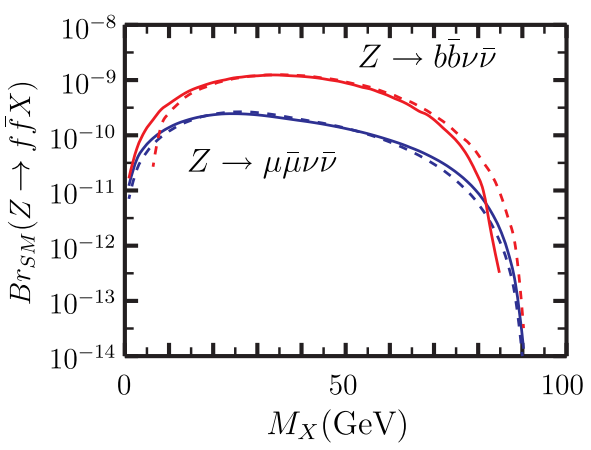

(a)

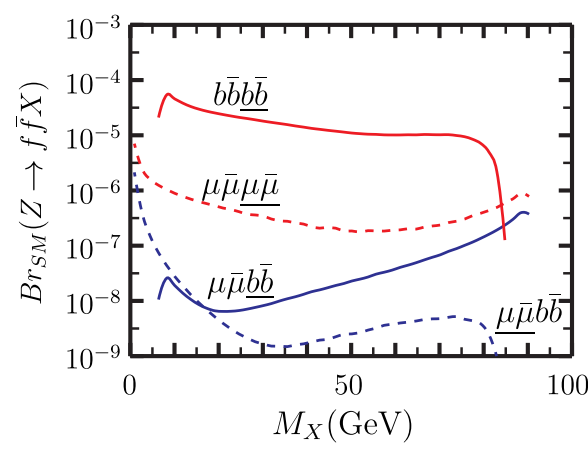

(b)

FIG. 10. (a) The SM background decay branching vs $M_{X}$. The solid/dash line is for the $X$ invisible/visible decay associated with $f=\mu, b$. (b) The SM background decay branching for visible decay associated with $\mu$ or $b$ vs $M_{X}$.

For quarkonium, $V_{Q} \rightarrow \bar{l}$ offers a clean tag for the particle identity and the di-lepton decay branching ratios, $\operatorname{Br}(J / \Psi \rightarrow$ $\bar{l})=11.932(46) \%$ and $\operatorname{Br}(\Upsilon \rightarrow l \bar{l})=7.46(15) \%[44]$ are well measured. Therefore, the dominant factor of the quarkonium identification is the detection efficiencies of di-lepton, and the overall $\xi$ will be similar to that of the four charged leptons final state. The expected $e(\mu)$ identification efficiency at the CEPC is $99.5 \%(98.5 \%)$ for charged lepton energy $E>10 \mathrm{GeV}$, and it drops to $\sim 96 \%(85 \%)$ when $E \sim$ $2 \mathrm{GeV}$ [2]. From these numbers, a simple estimation is that $\xi \sim(0.985)^{4}=0.94$ when all four muon energies are larger than $10 \mathrm{GeV}$. For the events with one muon energy around $2 \mathrm{GeV}$ and the other three $>10 \mathrm{GeV}, \xi \sim(0.85) \times(0.985)^{3}=$ 0.81 . For the $\mathrm{b}$ quark, the tagging efficiency is $\sim 90 \%$ [2]. ${ }^{2}$ Thus, $\xi \sim(0.9)^{4}=0.66$ for the $4 b$ channel. For the $\mu \bar{\mu} b \bar{b}$ channel, the overall detection efficiency ranges roughly from $\xi \sim 0.68$, with one low energy muon, to 0.78 when all particle energies are larger than $10 \mathrm{GeV}$. Of course, to determine the actual detection efficiency for a specific channel, a comprehensive analysis of the full kinematic and the detector performance is needed and we should leave it to the future studies.

As has been studied in $[1,23]$, the $Z \rightarrow S(b \bar{b}) f \bar{f}$ is a very promising channel for either discovery or falsifying a sizeable portion of the light scalar parameter space; see Fig. 9(a). This new $s_{\theta}^{2} \times \operatorname{Br}(S \rightarrow b \bar{b})$ limit outperforms the LEP-II limits [50] [the green curve in Fig. 9(a)] by around $5 / 1$ orders of magnitude at $M_{S}=10 / 80 \mathrm{GeV}$ by using the $Z \rightarrow \nu \bar{\nu} b \bar{b}$ channel due to the enormous number of $Z$ s that are expected to be produced. The signal will be a sharp b-pair invariant mass in the decay $Z \rightarrow \nu \bar{\nu} b \bar{b}$. This channel is useful for probing $S$ with large $b \bar{b}$ [or $\mu \bar{\mu}$ when $M_{S}<2 m_{b}$, Fig. 9(e)] decay fraction. A similar limit is

\footnotetext{
${ }^{2}$ At LEP, the efficiencies for b-tagging range from $21.0 \%$ to $44.0 \%$ depending on the b-purities [53]. With the implementation of new techniques like neural networks and boosted decision trees, an efficiency to identify $b$ jet of $70 \%$ can be achieved at the ATLAS [54].
}

obtained for the light vector; see Fig. 9(b). In the Higgs portal models, the Yukawa couplings of the singlet scalar are proportional to the fermion masses. Thus it is expected that the constraint on the mixing $s_{\theta}^{2}$ from the visible decay mode $S \rightarrow \mu \bar{\mu}$ is 4 orders of magnitude weaker than that of the $S \rightarrow b \bar{b}$ mode if the detector has similar energy resolutions on determining the invariant masses $m_{b \bar{b}}$ and $m_{\mu \bar{\mu}}$. On the other hand, the new vector boson couplings in general are not proportional to the fermion masses. Therefore, whether $V_{D} \rightarrow b \bar{b}$ or $V_{D} \rightarrow \mu \bar{\mu}$ are better visible channels for discovery will depend on the unknown detector performance in the future machine. The searching strategies we proposed to look for the vector boson with mass $1 \lesssim M_{V_{D}} \lesssim 80 \mathrm{GeV}$ at the $\mathrm{Z}$ factories is model independent. The $Z \rightarrow f \bar{f} V_{D}$ signals are determined by the phenomenological flavordependent gauge coupling $f_{D}^{f}$, assuming there is no mixing between the SM Z boson and the physical state $V_{D}$. On the other hand, the limits obtained in [51,55], where the kinematic mixing parameter can be probed down to $\epsilon \sim$ $10^{-3}$ by electroweak precision or the Drell-Yan processes at the LHC, requires a UV complete model. For instance, the Drell-Yan processes need the vector boson couplings to both quarks and leptons at the same time.

Moreover, we found that the invisible decay of $X$ can be utilized at the $\mathrm{Z}$ factory with precision controlled $e^{ \pm}$beam energy; see Fig. 9(c) and Fig. 9(d). This mode provides a powerful handle to probe the $X$ with sizable invisible decay branching ratios, for example the model discussed in [23]. We know of no other reactions that can compete with this for the mass range of $X$ we are studying. Furthermore, we have also studied the 2-body $Z \rightarrow \gamma X$ process with the production of back-to-back on-shell $X$ and a high-energy photon and found that the signal cannot compete with the SM background, $e^{+} e^{-} \rightarrow \gamma f \bar{f}$. However, with a higher center of mass energy, $\sqrt{s} \gtrsim 160 \mathrm{GeV}$, this $e^{+} e^{-} \rightarrow \gamma f \bar{f}$ channel is useful for detecting a vector boson with mass $\gtrsim 20 \mathrm{GeV}$ which kinematically mixes with the SM $U(1)_{Y}$ [52]. In this model, the $\operatorname{Br}\left(V_{D} \rightarrow f \bar{f}\right)$ is determined by the strength of the kinetic mixing parameter $\epsilon$. When 
$M_{X}<v_{H}$, the $X$-fermion coupling is mostly vectorlike and, therefore, $g_{D}^{f} \simeq \epsilon$. Their $2 \sigma$ limits on $\epsilon^{2}$ multiplied by $\operatorname{Br}\left(V_{D} \rightarrow \mu \bar{\mu}\right)$ for $f=\mu$ with $\mathcal{L}=1 \mathrm{ab}^{-1}$ at the FCC-ee $(160 \mathrm{GeV}, 350 \mathrm{GeV})$ and $\operatorname{CEPC}(240 \mathrm{GeV})$ are shown in Fig. 9(f). A different limit from the Drell-Yan process $p p \rightarrow V_{D} \rightarrow l l$ at LHC14 with $\mathcal{L}=3 \mathrm{ab}^{-1}$ [51] is displayed too. We stress that these limits apply solely to the specific $U(1)$ kinetic mixing model.

It is also worth pointing out that, since there is no meaningful SM background, the above-mentioned searches can set stringent constraints on the vector boson flavorchanging decay branching ratios $\operatorname{Br}\left(V_{D} \rightarrow f_{i} \bar{f}_{j}+f_{j} \bar{f}_{i}\right)$ as well. For example, by using the signal $Z \rightarrow \mu \bar{\mu} V_{D}\left(V_{D} \rightarrow\right.$ $\left.f_{i} \bar{f}_{j}+f_{j} \bar{f}_{i}\right)$, the combination $\left(g_{D}^{\mu}\right)^{2} \times \operatorname{Br}\left(V_{D} \rightarrow f_{i} \bar{f}_{j}+\right.$ $\left.f_{j} \bar{f}_{i}\right)$ can be probed to the $10^{-9}\left(10^{-3}\right)$ level for $M_{V_{D}} \sim$ a few (80) GeV. Additionally, in the case of an inert singlet scalar, which does not mix with the SM Higgs, the Z and Higgs factory cannot compete with the limit from the Higgs invisible decay $[48,49]$. We also considered the possibility of probing a light $X$ boson by utilizing the $Z \rightarrow V_{Q} X$ channel, where $V_{Q}=J / \Psi, \Upsilon$. Although the limits are relatively weak, this process provides an additional search strategy for $X$ with a large invisible (or $\mu \bar{\mu}$ ) decay branching ratio and a cross check if $X$ is found; see Figs. 9(c)-(f). On the other hand, the light exotic vector possibility will be excluded if the signal $Z \rightarrow \Upsilon \mu \bar{\mu}$ with a $\mu \bar{\mu}$ resonance is seen but the counterpart signal $Z \rightarrow b \bar{b} \mu \bar{\mu}$ is not.

Finally, we note that we can probe regions of parameter space with significantly smaller branching ratios to SM particles than has previously been considered. As such, it is worth the caution to check to see if the lifetime of the singlet scalar can ever be so long as to fake an invisible decay. We find that, for our range of masses, the mixing angle needs to be several orders of magnitude below what we consider before the singlet lifetime becomes an issue.

Of course, we cannot predict the details and parameters of the future machines. Our numerical estimations in this work should be regarded as explorative speculation at this moment. The realistic analysis is still awaited for the experimentalist to perform in the future.

\section{ACKNOWLEDGMENTS}

W. F. C. is supported by the Taiwan Minister of Science and Technology under Grants No. 106-2112-M-007-009MY3 and No. 105-2112-M-007-029. TRIUMF receives federal funding via a contribution agreement with the National Research Council of Canada and the Natural Science and Engineering Research Council of Canada.
[1] W.-F. Chang, T. Modak, and J. N. Ng, Signal for a light singlet scalar at the LHC, Phys. Rev. D 97, 055020 (2018).

[2] C.-S.S. Group, CEPC-SPPC Preliminary Conceptual Design Report. 1. Physics and Detector, http://www.lnf .infn.it/sis/frascatiseries/Volume61/Volume61.pdf.

[3] C.-S.S. Group, CEPC-SPPC Preliminary Conceptual Design Report. 2. Accelerator, http://www.lnf.infn.it/sis/ frascatiseries/Volume61/Volume61.pdf.

[4] M. Bicer et al. (TLEP Design Study Working Group collaboration), First look at the physics case of TLEP, J. High Energy Phys. 01 (2014) 164.

[5] D. d'Enterria, Physics at the FCC-ee, in Proceedings of 17th Lomonosov Conference on Elementary Particle Physics, (World Scientific, Moscow, Russia, 2017), p. 182.

[6] D. d'Enterria, Frascati Phys. Ser., in Proceedings LFC15: Physics Prospects for Linear and Other Future Colliders, ECT*, Trento, 2015, (INFN, Italy, 2016).

[7] J. Liu, L.-T. Wang, X.-P. Wang, and W. Xue, Exposing dark sector with future Z-factories, arXiv:1712.07237.

[8] N. Liu and L. Wu, An indirect probe of the higgsino world at the CEPC, Eur. Phys. J. C 77, 868 (2017).

[9] P. Huang, A. J. Long, and L.-T. Wang, Probing the electroweak phase transition with Higgs factories and gravitational waves, Phys. Rev. D 94, 075008 (2016).

[10] W.-F. Chang, J. N. Ng, and J. M. S. Wu, Constraints on new scalars from the LHC $125 \mathrm{GeV}$ Higgs signal, Phys. Rev. D 86, 033003 (2012).
[11] J. N. Ng and A. de la Puente, Electroweak vacuum stability and the seesaw mechanism revisited, Eur. Phys. J. C 76, 122 (2016).

[12] U. Ellwanger, The next-to-minimal supersymmetric standard model, in Proceedings of 45th Rencontres de Moriond on Electroweak Interactions and Unified Theories: La Thuile, Italy, 2010 (Moriond, Paris, France, 2010), p. 89.

[13] P. Athron, S. F. King, D. J. Miller, S. Moretti, and R. Nevzorov, The constrained exceptional supersymmetric standard model, Phys. Rev. D 80, 035009 (2009).

[14] C. Balzs, A. Mazumdar, E. Pukartas, and G. White, Baryogenesis, dark matter and inflation in the next-to-minimal supersymmetric standard model, J. High Energy Phys. 01 (2014) 073.

[15] S. Akula, C. Balzs, L. Dunn, and G. White, Electroweak baryogenesis in the $\mathbb{Z}_{3}$-invariant NMSSM, J. High Energy Phys. 11 (2017) 051.

[16] I. P. Ivanov, Building and testing models with extended Higgs sectors, Prog. Part. Nucl. Phys. 95, 160 (2017).

[17] M. Fink and H. Neufeld, Neutrino masses in a conformal multi-Higgs-doublet model, arXiv:1801.10104.

[18] T. Vieu, A. P. Morais, and R. Pasechnik, Electroweak phase transitions in multi-Higgs models: the case of Trinificationinspired THDSM, arXiv:1801.02670.

[19] D. Croon, V. Sanz, and E. R. M. Tarrant, Reheating with a composite Higgs boson, Phys. Rev. D 94, 045010 (2016). 
[20] U. Aydemir, D. Minic, C. Sun, and T. Takeuchi, Pati-Salam unification from noncommutative geometry and the TeVscale $W_{R}$ boson, Int. J. Mod. Phys. A 31, 1550223 (2016).

[21] U. Aydemir, D. Minic, C. Sun, and T. Takeuchi, The $750 \mathrm{GeV}$ diphoton excess in unified $S U(2)_{L} \times S U(2)_{R} \times$ $S U(4)$ models from noncommutative geometry, Mod. Phys. Lett. A 31, 1650101 (2016).

[22] D. Croon, B. M. Dillon, S. J. Huber, and V. Sanz, Exploring holographic composite Higgs models, J. High Energy Phys. 07 (2016) 072.

[23] W.-F. Chang and J. N. Ng, Renormalization group study of the minimal majoronic dark radiation and dark matter model, J. Cosmol. Astropart. Phys. 07 (2016) 027.

[24] W.-F. Chang and J. N. Ng, Minimal model of Majoronic dark radiation and dark matter, Phys. Rev. D 90, 065034 (2014).

[25] L. G. Cabral-Rosetti, R. Gaitn, J. H. M. de Oca, R. O. Galicia, and E. A. Garcs, Scalar dark matter in inert doublet model with scalar singlet, J. Phys. Conf. Ser. 912, 012047 (2017).

[26] I. Garg, S. Goswami, V. K. N., and N. Khan, Electroweak vacuum stability in presence of singlet scalar dark matter in TeV scale seesaw models, Phys. Rev. D 96, 055020 (2017).

[27] J. McKay (GAMBIT Collaboration), Global fits of the scalar singlet model using GAMBIT, in Proceedings of 2017 European Physical Society Conference on High Energy Physics, Venice, Italy, July 5-12, 2017, Proc. Sci., EPS-HEP2017 (2017) 076.

[28] P. Athron et al. (GAMBIT Collaboration), Status of the scalar singlet dark matter model, Eur. Phys. J. C 77, 568 (2017).

[29] C. P. Burgess, M. Pospelov, and T. t. Veldhuis, The minimal model of nonbaryonic dark matter: A singlet scalar, Nucl. Phys. B619, 709 (2001).

[30] J. McDonald, Gauge singlet scalars as cold dark matter, Phys. Rev. D 50, 3637 (1994).

[31] J. M. Cline, K. Kainulainen, P. Scott, and C. Weniger, Update on scalar singlet dark matter, Phys. Rev. D 88, 055025 (2013).

[32] S. Profumo, M. J. Ramsey-Musolf, C. L. Wainwright, and P. Winslow, Singlet-catalyzed electroweak phase transitions and precision Higgs boson studies, Phys. Rev. D 91, 035018 (2015).

[33] S. Profumo, M. J. Ramsey-Musolf, and G. Shaughnessy, Singlet Higgs phenomenology and the electroweak phase transition, J. High Energy Phys. 08 (2007) 010.

[34] G. A. White, A Pedagogical Introduction to Electroweak Baryogenesis, IOP Concise Physics (Morgan and Claypool, London, 2016).

[35] J. Kozaczuk, S. Profumo, L. S. Haskins, and C. L. Wainwright, Cosmological phase transitions and their properties in the NMSSM, J. High Energy Phys. 01 (2015) 144.

[36] J. Elias-Miro, J. R. Espinosa, G. F. Giudice, H. M. Lee, and A. Strumia, Stabilization of the electroweak vacuum by a scalar threshold effect, J. High Energy Phys. 06 (2012) 031 .
[37] M. Gonderinger, Y. Li, H. Patel, and M. J. Ramsey-Musolf, Vacuum stability, perturbativity, and scalar singlet dark matter, J. High Energy Phys. 01 (2010) 053.

[38] M. Gonderinger, H. Lim, and M. J. Ramsey-Musolf, Complex scalar singlet dark matter: Vacuum stability and phenomenology, Phys. Rev. D 86, 043511 (2012).

[39] N. Khan and S. Rakshit, Study of electroweak vacuum metastability with a singlet scalar dark matter, Phys. Rev. D 90, 113008 (2014).

[40] C. Balazs, A. Fowlie, A. Mazumdar, and G. White, Gravitational waves at aLIGO and vacuum stability with a scalar singlet extension of the Standard Model, Phys. Rev. D 95, 043505 (2017).

[41] A. Belyaev, N. D. Christensen, and A. Pukhov, CalcHEP 3.4 for collider physics within and beyond the Standard Model, Comput. Phys. Commun. 184, 1729 (2013).

[42] W.-F. Chang, J. N. Ng, and J. M. S. Wu, A very narrow shadow extra Z-boson at colliders, Phys. Rev. D 74, 095005 (2006).

[43] W.-F. Chang, J. N. Ng, and J. M. S. Wu, Shadow Higgs from a scale-invariant hidden U(1)(s) model, Phys. Rev. D 75, 115016 (2007).

[44] C. Patrignani et al. (Particle Data Group Collaboration), Review of particle physics, Chin. Phys. C 40, 100001 (2016).

[45] B. Guberina, J. H. Kuhn, R. D. Peccei, and R. Ruckl, Rare decays of the Z0, Nucl. Phys. B174, 317 (1980).

[46] T.-C. Huang and F. Petriello, Rare exclusive decays of the Z-boson revisited, Phys. Rev. D 92, 014007 (2015).

[47] G. T. Bodwin, H. S. Chung, J.-H. Ee, and J. Lee, Z-boson decays to a vector quarkonium plus a photon, Phys. Rev. D 97, 016009 (2018).

[48] G. Aad et al. (ATLAS Collaboration), Search for invisible decays of a Higgs boson using vector-boson fusion in $p p$ collisions at $\sqrt{s}=8 \mathrm{TeV}$ with the ATLAS detector, J. High Energy Phys. 01 (2016) 172.

[49] CMS Collaboration, A combination of searches for the invisible decays of the Higgs boson using the CMS detector, Report No. CMS-PAS-HIG-15-012, 2015.

[50] R. Barate et al. (OPAL, DELPHI, LEP Working Group for Higgs boson searches, ALEPH, L3 Collaborations), Search for the standard model Higgs boson at LEP, Phys. Lett. B 565, 61 (2003).

[51] D. Curtin, R. Essig, S. Gori, and J. Shelton, Illuminating dark photons with high-energy colliders, J. High Energy Phys. 02 (2015) 157.

[52] M. He, X.-G. He, C.-K. Huang, and G. Li, Search for a heavy dark photon at future $e^{+} e^{-}$colliders, J. High Energy Phys. 03 (2018) 139.

[53] J. Abdallah et al. (DELPHI Collaboration), b-tagging in DELPHI at LEP, Eur. Phys. J. C 32, 185 (2004).

[54] G. Aad et al. (ATLAS Collaboration), Performance of $b$-Jet identification in the ATLAS experiment, J. Instrum. 11, P05013 (2016).

[55] I. Hoenig, G. Samach, and D. Tucker-Smith, Searching for dilepton resonances below the $\mathrm{Z}$ mass at the LHC, Phys. Rev. D 90, 075016 (2014). 